\title{
GENE ACTION AND COMBINING ABILITY FOR GRAIN YIELD AND YIELD RELATED TRAITS IN RICE (Oryza sativa L.)
}

\author{
H. Dorosti ${ }^{1}$, S. Monajjem ${ }^{2}$
}

\begin{abstract}
Line (five) $\times$ tester (two) analysis was conducted to determine good parents and the nature of gene action governing yield and its component traits in rice. The line $\times$ testers showed significant differences for all traits except for number of spikelets per panicle and hundred grain weight. Parents with low $\times$ high general combining ability (GCA) effects resulted in heterotic hybrid combination like the IR62829A/IR57301-158-1R. A low $\times$ low GCA gave the best heterotic combination i.e. IR68888A/'Sepidroud'. The study showed GCA to only identify better parental lots and it will be unwise to discard the low GCA types. Predominance of dominance gene action for all the traits studied except for flag leaf width and number of spikelets per panicle suggests heterosis breeding must be strongly pursued for exploitation of the yield advantage over the inbred varieties.
\end{abstract}

Keywords: combining ability, cytoplasmic male sterility, gene action, hybrid rice, yield

\section{INTRODUCTION:}

Hybrid rice technology assures the rice farmers with increased yields over improved conventional varieties by 15 to $20 \%$ (Tran, 2002) thereby enhanced field incomes. Hybrid rice area is now rapidly increasing outside China ever since the release of the first set of hybrids in China in 1976 (Yaun, 1998). Presently, many countries other than China like Vietnam, India, Indonesia, Iran, Philippines, United States of America, Bangladesh, Sri Lanka, Myanmar and Egypt are currently engaged in developing hybrid rice technology to suit their local conditions. Area under hybrid rice cultivation outside China has substantially increased beyond the 1 million ha mark in 2003 due to active collaboration with the public and private institutions (Virmani and Kumar, 2004). Efforts are underway in many countries including Iran to develop hybrid combinations and to sustain the hybrid technology suitable for local environmental conditions (Allahgholipour and Ali, 2006). Magnitude of heterosis, grain quality and resistance to biotic stresses need to be addressed for successful deployment of hybrid technology. Similarly adaptability of the parental lines and their overall performance for grain quality, and resistance to insect and pests also needs to be assessed to develop superior hybrid combinations (Allahgholipour and Ali, 2006). In Iran the hybrid rice technology is already bearing fruits with the release of IRH1 in over 130ha in the year 2006 and plans to increase the area to 2000 ha in 2007 with an average yield performance of 9.0 tonnes/ha as compared to Khazar an improved variety with only $6 \mathrm{t} / \mathrm{ha}$ (Ali, 2006). However, for the search of newer hybrid combinations with better quality features it is important to study the genetic nature and combining ability of the parents that are going to be involved in hybrid combinations. Good combiner parents often result in higher frequency of heterotic hybrids than poor combiner parents. However, in the recent study by Allahgholipour and Ali (2006) found that low GCA x low GCA crosses can give heterotic combinations and will be unwise to discard the low GCA parental types. 
Selection of appropriate parents by studying their combining ability and heterosis still remains best option for increasing the breeding efficiency in identifying heterotic hybrids. By analyzing the combining ability and estimating the degree of heterosis, clues on the nature of gene action, desirable parents and important yield traits may be found (Can et al., 1997). Hybrid rice breeding programs in different countries used combining ability, gene action and heterosis studies for identifying suitable parents for local needs (Gravois and Mc new, 1993; Moon et al., 1994; Siddiq et al., 1994). GCA and SCA effects are useful for hybrid rice breeding program by identifying traits that are predominantly governed by non- additive genetic variance such as number of panicles per plant, number of spikelets per panicle, test grain weight, total dry matter accumulation, spikelet fertility and grain yield (Siddiq et al., 1994). Several researchers have already studied the combining ability and gene action on various traits including yield. However, there is scarce information is available under north Iranian conditions which has temperate conditions with longer photoperiods. Therefore, a study was carried out to obtain information on the combining ability and the gene action of locally adaptable restorers and cytoplasmic male sterile (CMS) lines for heterosis breeding towards rice production and productivity enhancement in Iran.

\section{MATERIALS AND METHODS}

Two stable CMS lines i.e. IR68888A and IR62829A and 5 restorer lines i.e. Sepidroud, Bijar, IR28, IR57301-158-1R, IR59673-93-23-3R were selected as parental tester and lines respectively. To determine GCA and SCA, the CMS lines IR68888A and IR62829A as testers were crossed with 5 restorer lines i.e. Sepidroud, Bijar, IR28, IR57301-158-1R, and IR59673-93-2-3-3R to generate 10 hybrid combinations in line $\times$ tester mating design (Kumar and Saini, 1981). The hybrids were evaluated along with parents in a randomized complete block design with three replications at Rice Research Institute of Iran (RRII) in 2013. Standard agronomic and irrigation management practices were adopted for proper growth and development of the hybrids and their parents. The isogenic B lines were raised in place of $\mathrm{A}$ lines for recording the grain yield and related component traits. The grain yield (GY) was measured. The other yield related traits Flag leaf length (FLL); Flag leaf width (FLW); Flag leaf area (FLA); Number of tillers (NT); Plant Height (PH); Panicle length (PL); Number of spikelets per panicle (NSP); Hundred grain weight (HGW); percent spikelet fertility (PSF) were all measured according to the standard evaluation system (IRRI, 1996). GCA of lines (restorers) and testers (CMS) and SCA of progenies were calculated (Kempthorne, 1957). For effective mean comparison of yield and its component traits for the parents and the hybrids were compared using Duncan's multiple range test (DMRT) (Gomez and Gomez, 1993). Midparent heterosis (MPH) was determined as percentage increase of the $F_{1}$ over the mean parental value for a given trait (Virmani, et al.,1997).

\section{RESULTS AND DISCUSSION}

Analysis of variance (ANOVA) for combining ability revealed significant differences between the treatments (genotype) for all the traits studied. Amongst the treatments the MSS due to parents showed significant differences for all the traits except the grain yield (Table 01). Also the MSS due to crosses was highly significant for all the traits, except for PL. The MSS due to parents versus crosses also differed significantly, except for FLW, FLA, NT, and HGW. The lines (restorers) showed significant differences to all the traits except PL. However, the testers showed significant differences for all traits except PL, HGW, and GY. The ANOVA revealed significant differences for all the traits for MSS due to line $\times$ tester except for NSP and HGW. 
An estimation of the general combining ability (GCA) effect of testers was found to be non- significant for GY, HGW, PL and FLW. Amongst the five parental lines three lines i.e. IR28, IR57301-158-1R, Bijar showed significantly positive GCA effects for GY and PSF. Bijar also showed significant positive effects for HGW, PL, PH while IR28 showed for NSP and PH. Likewise, IR57301-158-1R gave significant positive GCA effects for FLL and FLA. IR28 was identified as good combiner for GY, PSF, and NSP. IR57301$158-1 \mathrm{R}$ was determined as a good combiner for several traits like showed for GY, PSF, FLL and FLA (Table 02).

Table 01: Analysis of variance for yield and yield related traits in line $x$ tester design

\begin{tabular}{lcrrrrrrrrrr}
\hline $\begin{array}{l}\text { Source of } \\
\text { Variance }\end{array}$ & df & $\begin{array}{r}\text { FLL } \\
(\mathrm{cm})\end{array}$ & $\begin{array}{r}\text { FLW } \\
(\mathrm{mm})\end{array}$ & $\begin{array}{r}\text { FLA } \\
\left(\mathrm{cm}^{2}\right)\end{array}$ & $\begin{array}{c}\text { NT } \\
(\mathrm{nos})\end{array}$ & $\begin{array}{r}\text { PH } \\
(\mathrm{cm})\end{array}$ & $\begin{array}{r}\text { PL } \\
(\mathrm{cm})\end{array}$ & $\begin{array}{r}\text { NSP } \\
(\mathrm{nos} .)\end{array}$ & $\begin{array}{r}\text { PSF } \\
(\%)\end{array}$ & $\begin{array}{c}\text { HGW } \\
(\mathrm{g})\end{array}$ & $\begin{array}{c}\text { GY } \\
(\mathrm{Kg} / \mathrm{ha})\end{array}$ \\
\hline Replication & 2 & 173 & 0.003 & 3.97 & 6.29 & 0.04 & 0.21 & 1579.5 & 14.72 & 0.001 & $1526.73^{\text {ns }}$ \\
Treatment & 16 & $28.19^{* *}$ & $0.097^{* *}$ & $32.02^{* *}$ & $47.30^{* *}$ & $258.54^{* *}$ & $6.19^{* *}$ & $3945.59^{* *}$ & $1229.81^{* *}$ & $0.328^{* *}$ & $11117.12^{* *}$ \\
parent & 6 & $58.33^{* *}$ & $4.960^{* *}$ & $64.08^{* *}$ & $68.51^{* *}$ & $365.15^{* *}$ & $9.63^{* *}$ & $2244.92^{* *}$ & $221.98^{* *}$ & $0.631^{* *}$ & $2158.90^{\text {ns }}$ \\
cross & 9 & $11.18^{* *}$ & $0.007^{* *}$ & $13.94^{* *}$ & $84.08^{* *}$ & $106.04^{* *}$ & $1.99^{\text {ns }}$ & $4710.29^{* *}$ & $1626.27^{* *}$ & $0.163^{* *}$ & $14822.11^{* *}$ \\
parent x cross & 1 & $14480.1^{* *}$ & $0.008^{\text {ns }}$ & $2.31^{\text {ns }}$ & $5.99^{\text {ns }}$ & $991.5^{* *}$ & $32.2^{* *}$ & $7260.3^{* *}$ & $9080.2^{* *}$ & $0.007^{\text {ns }}$ & $31521.5^{* *}$ \\
Line & 4 & $6.60^{*}$ & $0.008^{* *}$ & $6.78^{* *}$ & $60.43^{* *}$ & $177.44^{* *}$ & $1.80^{\text {ns }}$ & $8687.91^{* *}$ & $3421.34^{* *}$ & $0.338^{* *}$ & $27189.89^{* *}$ \\
Tester & 1 & $57.68^{* *}$ & $0.01^{*}$ & $55.08^{* *}$ & $174.96^{* *}$ & $142.35^{* *}$ & $0.39^{\text {ns }}$ & $6109.84^{* *}$ & $510.38^{* *}$ & $0.009^{\text {ns }}$ & $580.44^{\text {ns }}$ \\
Line x Tester & 4 & $4.13^{* *}$ & $0.007^{* *}$ & $10.82^{* *}$ & $850.15^{* *}$ & $25.55^{* *}$ & $2.59^{*}$ & $382.78^{\text {ns }}$ & $27846.46^{* *}$ & $.012^{\text {ns }}$ & $6014.74^{* *}$ \\
Error & 32 & 2.40 & 0.002 & 1.50 & 16.71 & 4.66 & 0.72 & 325.49 & 21.57 & 0.008 & 57.90 \\
\hline
\end{tabular}

FLL=Flag leaf length; FLW=Flag leaf width; FLA=Flag leaf area; NT=Number of tillers; $P H=$ Plant Height; $\mathrm{PL}=$ Panicle length; $\mathrm{NSP}=\mathrm{Number}$ of spikelets per panicle; $\mathrm{HGW}=$ Hundred grain weight; $\mathrm{PSF}=$ percent spikelet fertility; GY=Grain yield

*,** Significant at 5 and $1 \%$ probability levels respectively and ns= non- significant.

Table 02: General combining ability (GCA) effects of lines and testers for rice grain yield and yield

\begin{tabular}{|c|c|c|c|c|c|c|c|c|c|c|}
\hline Genotype & $\begin{array}{l}\text { FLL } \\
(\mathrm{cm})\end{array}$ & $\begin{array}{l}\text { FLW } \\
(\mathrm{mm})\end{array}$ & $\begin{array}{l}\text { FLA } \\
\left(\mathrm{cm}^{2}\right)\end{array}$ & $\begin{array}{c}\text { NT } \\
\text { (nos.) }\end{array}$ & $\begin{array}{l}\mathrm{PH} \\
(\mathrm{cm})\end{array}$ & $\begin{array}{l}\text { PL } \\
(\mathrm{cm})\end{array}$ & $\begin{array}{l}\text { NSP } \\
\text { (nos.) }\end{array}$ & $\begin{array}{l}\text { PSF } \\
(\%)\end{array}$ & $\begin{array}{c}\mathrm{HGW} \\
(\mathrm{g})\end{array}$ & $\begin{array}{c}\text { GY } \\
(\mathrm{Kg} / \mathrm{ha})\end{array}$ \\
\hline \multicolumn{11}{|l|}{ Lines } \\
\hline Sepidroud & $0.075^{\mathrm{ns}}$ & $0.052^{\mathrm{ns}}$ & $0.37^{\mathrm{ns}}$ & $5.248^{*}$ & $0.48^{\mathrm{ns}}$ & 0.31 & $2.05^{\mathrm{ns}}$ & $10.31^{* *}$ & $0.202^{* *}$ & $-2.578^{\mathrm{ns}}$ \\
\hline Bijar & $-1.34^{*}$ & $0.049^{\mathrm{ns}}$ & $0.80^{\mathrm{ns}}$ & $1.794^{\mathrm{ns}}$ & $3.54^{*}$ & $0.76^{*}$ & $-0.70^{\mathrm{ns}}$ & $3.94^{* *}$ & $0.264^{* *}$ & $29.532^{*}$ \\
\hline IR28 & $-0.70^{\text {ns }}$ & $-0.014^{\mathrm{ns}}$ & $-1.32^{*}$ & $-0.794^{\text {ns }}$ & $3.54^{*}$ & $-0.65^{\text {ns }}$ & $33.08^{*}$ & $14.15^{* *}$ & $0.005^{\text {ns }}$ & $52.074^{* *}$ \\
\hline IR57301-158-1R & $1.27^{*}$ & $0.014^{\mathrm{ns}}$ & $1.34^{*}$ & $1.863^{\mathrm{ns}}$ & $1.87^{\mathrm{ns}}$ & $-0.23^{\text {ns }}$ & $28.05^{\text {ns }}$ & $13.67^{* *}$ & $-0.162^{* *}$ & $36.03^{*}$ \\
\hline IR59673-2-3-3R & $07^{\mathrm{ns}}$ & $0.004^{\text {ns }}$ & $0.41^{\mathrm{ns}}$ & $2.366^{\mathrm{ns}}$ & $-9.45^{* *}$ & $-0.18^{\text {ns }}$ & $-62.49^{\text {ns }}$ & $-42.08^{* *}$ & $-0.298^{* *}$ & $-11.057^{* *}$ \\
\hline SE & 63 & 0.018 & 0.50 & 1.67 & 0.88 & 0.34 & 7.36 & 1.89 & 0.036 & 9.788 \\
\hline \multicolumn{11}{|l|}{ Tester } \\
\hline IR68888A & $1.38^{* *}$ & $0.019^{\mathrm{ns}}$ & $1.35^{* *}$ & $2.415^{*}$ & $2.17^{* *}$ & $-0.11^{\mathrm{ns}}$ & $14.27^{* *}$ & $4.12^{* *}$ & $-0.018^{\mathrm{ns}}$ & $-4.399^{\text {ns }}$ \\
\hline IR62829A & $1.38^{* *}$ & $0.018^{\mathrm{ns}}$ & $-1.35^{* *}$ & $2.415^{*}$ & $-2.17^{* *}$ & $0.11^{\mathrm{ns}}$ & $-14.27^{* *}$ & $-4.12^{* *}$ & $0.018^{\mathrm{ns}}$ & $4.399^{\mathrm{ns}}$ \\
\hline SE & 0.4 & 0.011 & 0.31 & 1.056 & 0.55 & 0.21 & 4.65 & 1.19 & 0.023 & 6.191 \\
\hline
\end{tabular}

FLL=Flag leaf length; FLW=Flag leaf width; FLA=Flag leaf area; NT=Number of tillers; PH=Plant Height; $\mathrm{PL}=$ Panicle length; $\mathrm{NSP}=$ Number of spikelets per panicle; $\mathrm{HGW}=$ Hundred grain weight; $\mathrm{PSF}=$ percent spikelet fertility; GY $=$ Grain yield

$*, * *$ Significant at 5 and $1 \%$ probability levels respectively and ns= non- significant. 
However, the Iranian landrace Bijar was found to be good combiner for GY, HGW, PSF and PL and can be effectively utilized with other male sterile lines and also in restorer breeding programs. Two combinations i.e. IR68888A/ Sepidroud and IR62829A/ IR57301-1581R showed significant SCA effects for grain yield. However, IR68888A/Sepidroud showed significantly positive SCA effects for GY and NT and negative SCA effects for FLA. Only IR62829A/Sepidroud showed significant difference in SCA effect for increased FLA. Out of ten hybrid combinations as many as six hybrid combinations showed non-significant differences for all the traits studied. All the hybrids showed non significant SCA effects for nearly seven of the ten traits studied i.e. FLL, FLW, PH, PL, NSP, PSF and HGW (Table 03).

Mean comparison of grain yield and its component traits using DMRT analysis revealed that IR68888A/Sepidroud hybrid combination performed significantly better than the other hybrids and parents for almost all the traits except for FLL, FLW and FLA. Further, as many as three hybrids performed significantly better than the parents for grain yield (Table 04). However, the testers in this study recorded a poor performance for most of the traits including the grain yield except for PL, NT, and PSF. Amongst the parental lines the 'Sepidroud' gave the highest mean grain yield $5135 \mathrm{~kg} / \mathrm{ha}$, FLL $(29.95 \mathrm{~cm})$ and PL $(27.12 \mathrm{~cm})$.

Mid-parent heterosis (MPH) for grain yield was positive ranging from 15 to $38.9 \%$ for five out of ten hybrids. Amongst them two hybrid combinations i.e. IR68888A/ Sepidroud and IR62829A/IR57301-158-1R had MPH for yield as 38.9 and $28.4 \%$ with corresponding actual yield levels of 5969 and $5518 \mathrm{~kg} /$ ha respectively (Table 04 and 05). Likewise the same two combinations were also detected by highly significant positive SCA combinations for grain yield (Table 3 ). The hybrid combination IR62829A/IR57301-158-
1R gave positive MPH for GY (28.4\%), NT (1.5\%), FLA (9.6\%), PH (0.5\%) and HGW $(12.8 \%)$ besides a negative heterobeltiosis for PSF (-14.8\%), FLL (-18.2\%) and PL ($4.2 \%$ ) (Table 05 ). All ten hybrid combinations showed positive MPH for HGW (ranging from 1.3 to $26.3 \%$ ) while seven hybrid combinations showed negative MPH for FLA (-4.9 to $-39.0 \%)$ and PL (-0.1 to $-11.9 \%$ ) (Table 05). However, the hybrid combination IR62829A/ IR59673-73-3R with a highest positive MPH for FLL (29.5\%), PSF (34.8\%) and PL (1.2\%) gave the lowest MPH for GY (-57\%) (Table 05). Components of genetic variance revealed predominance of dominant component of variance for all the traits except for $\mathrm{HGW}$ and PH (Table 06). Heritability (narrow sense) for NSP, HGW, PSF and PH were 36.7, 33.7, 30.2 and $24.2 \%$, respectively. Most influenced trait by environment was FLL (73.7\%) and with a low heritability (narrow sense) of $9.83 \%$ (Table 06).

In the present study, the ANOVA for combining ability revealed significant differences between the treatments (genotype) for all the traits studied and amongst the treatments showed significant differences for all the traits except grain yield. FLL, FLW, FLA, NT, PH and PSF showed significant differences for the lines, testers, line $\times$ testers. Selected lines were significantly different for all the traits except for PL indicating a better representation and selection for this study. However, only two testers (CMS lines) were significantly different for all the traits except for 205 grain yield, HGW and PL.

The tester parents IR68888A and IR62829A to be poor combiners for four (FLW, PL, HGW and GY) of the ten traits under study indicated the need for developing adaptable and stable CMS lines with high GCA to suit the present local conditions. The testers probably revealed different genetic backgrounds based on the ANOVA and GCA effects. However, these two CMS lines were stably sterile under Rasht, Iran conditions. 
Table 03: Specific combining ability (SCA) effects for grain yield and yield related traits of ten hybrid combinations

\begin{tabular}{|c|c|c|c|c|c|c|c|c|c|c|}
\hline Genotype & $\begin{array}{l}\text { FLL } \\
(\mathrm{cm})\end{array}$ & $\begin{array}{l}\text { FLW } \\
(\mathrm{mm})\end{array}$ & $\begin{array}{l}\text { FLA } \\
\left(\mathrm{cm}^{2}\right)\end{array}$ & $\begin{array}{c}\text { NT } \\
\text { (nos.) }\end{array}$ & $\begin{array}{c}\mathrm{PH} \\
(\mathrm{cm})\end{array}$ & $\begin{array}{c}\mathrm{PL} \\
(\mathrm{cm})\end{array}$ & $\begin{array}{l}\text { NSP } \\
\text { (nos.) }\end{array}$ & $\begin{array}{l}\text { PSF } \\
(\%)\end{array}$ & $\begin{array}{c}\text { HGW } \\
\text { (g) }\end{array}$ & $\begin{array}{c}\text { GY } \\
(\mathrm{Kg} / \mathrm{ha})\end{array}$ \\
\hline IR68888A / Sepidroud & $-0.75^{\mathrm{ns}}$ & $-0.019^{\mathrm{ns}}$ & $-2.28^{*}$ & $5.64^{*}$ & $0.31^{\mathrm{ns}}$ & $-0.21^{\mathrm{ns}}$ & $5.86^{\mathrm{ns}}$ & $5.84^{\mathrm{ns}}$ & $-0.006^{\mathrm{ns}}$ & $50.63^{*}$ \\
\hline IR62829A/ Sepidroud & $0.75^{\mathrm{ns}}$ & $0.019^{\text {ns }}$ & $2.28^{*}$ & $-5.64^{*}$ & $-0.31^{\text {ns }}$ & $0.21^{\mathrm{ns}}$ & $-5.86^{\text {ns }}$ & $-5.84^{\text {ns }}$ & $0.006^{\mathrm{ns}}$ & $-50.63^{*}$ \\
\hline IR68888A / Bijar & $-0.17^{\text {ns }}$ & $0.035^{\mathrm{ns}}$ & $0.64^{\mathrm{ns}}$ & $2.12^{\mathrm{ns}}$ & $-0.10^{\text {ns }}$ & $0.17^{\mathrm{ns}}$ & $-13.17^{\mathrm{ns}}$ & $-4.92^{\text {ns }}$ & $0.110^{\mathrm{ns}}$ & $-7.48^{\mathrm{ns}}$ \\
\hline IR62829A/ Bijar & $0.17^{\mathrm{ns}}$ & $-0.035^{\mathrm{ns}}$ & $-0.64^{\mathrm{ns}}$ & $-2.12^{\mathrm{ns}}$ & $0.10^{\mathrm{ns}}$ & $-0.17^{\mathrm{ns}}$ & $13.17^{\mathrm{ns}}$ & $4.92^{\text {ns }}$ & $-0.110^{\text {ns }}$ & $7.48^{\mathrm{ns}}$ \\
\hline IR68888A/ IR28 & $-0.80^{\text {ns }}$ & $0.032^{\mathrm{ns}}$ & $0.04^{\mathrm{ns}}$ & $-3.25^{\mathrm{ns}}$ & $-1.12^{\mathrm{ns}}$ & $1.03^{\mathrm{ns}}$ & $-2.10^{\mathrm{ns}}$ & $1.21^{\mathrm{ns}}$ & $0.076^{\mathrm{ns}}$ & $-10.35^{\mathrm{ns}}$ \\
\hline IR62829A / IR28 & $0.80^{\mathrm{ns}}$ & $-0.032^{\mathrm{ns}}$ & $-0.04^{\text {ns }}$ & $3.25^{\mathrm{ns}}$ & $1.12^{\mathrm{ns}}$ & $-1.03^{\text {ns }}$ & $2.10^{\text {ns }}$ & $-1.21^{\mathrm{ns}}$ & $-0.076^{\mathrm{ns}}$ & $10.35^{\text {ns }}$ \\
\hline $\begin{array}{l}\text { IR68888A / IR57301- } \\
\text { 158-1R }\end{array}$ & $0.94^{\mathrm{ns}}$ & $-0.003^{\mathrm{ns}}$ & $1.19^{\mathrm{ns}}$ & $-2.75^{\mathrm{ns}}$ & $3.54^{\mathrm{ns}}$ & $-0.27^{\mathrm{ns}}$ & $5.12^{\text {ns }}$ & $-3.23^{\mathrm{ns}}$ & $-0.009^{n s}$ & $-35.69^{*}$ \\
\hline $\begin{array}{l}\text { IR62829A / IR57301- } \\
\text { 158-1R }\end{array}$ & $-0.94^{\text {ns }}$ & $0.003^{\mathrm{ns}}$ & $-1.19^{\text {ns }}$ & $2.75^{\mathrm{ns}}$ & $-3.45^{\text {ns }}$ & $0.27^{\mathrm{ns}}$ & $-5.12^{\mathrm{ns}}$ & $3.23^{\mathrm{ns}}$ & $.0009^{\mathrm{ns}}$ & $35.69^{*}$ \\
\hline $\begin{array}{l}\text { IR68888A / IR59673-2- } \\
\text { 3-3R }\end{array}$ & $0.79^{\mathrm{ns}}$ & $-0.044^{\mathrm{ns}}$ & $0.40^{\mathrm{ns}}$ & $-1.69^{\text {ns }}$ & $-1.34^{\mathrm{ns}}$ & $-0.71^{\mathrm{ns}}$ & $0.67^{\mathrm{ns}}$ & $1.28^{\mathrm{ns}}$ & $-0.018^{\mathrm{ns}}$ & $2.89^{\mathrm{ns}}$ \\
\hline $\begin{array}{l}\text { IR62829A / IR59673-2- } \\
\text { 3-3R }\end{array}$ & $-0.79^{\text {ns }}$ & $0.04^{\mathrm{ns}}$ & $-0.40^{\text {ns }}$ & $1.69^{\mathrm{ns}}$ & $1.34^{\mathrm{ns}}$ & $0.71^{\mathrm{ns}}$ & $-0.67^{\mathrm{ns}}$ & $-1.28^{\mathrm{ns}}$ & $0.018^{\mathrm{ns}}$ & $-2.89^{\text {ns }}$ \\
\hline S.E & 0.89 & 0.02 & 0.70 & 2.36 & 1.55 & 0.49 & 10.42 & 2.68 & 0.05 & 13.84 \\
\hline
\end{tabular}

FLL=Flag leaf length; FLW=Flag leaf width; FLA=Flag leaf area; NT=Number of tillers; PH=Plant Height; PL=Panicle length; NSP=Number of spikelets per panicle; $\mathrm{HGW}=$ Hundred grain weight; $\mathrm{PSF}=$ percent spikelet fertility; GY=Grain yield; *,** Significant at 5 and $1 \%$ probability levels respectively and $n s=$ non- significant.

Table 04: Mean comparison of grain yield and yield related traits in hybrids and their parents

\begin{tabular}{|c|c|c|c|c|c|c|c|c|c|}
\hline Crosses & $\begin{array}{l}\text { FLL } \\
(\mathrm{cm})\end{array}$ & $\begin{array}{l}\text { FLW } \\
(\mathrm{mm})\end{array}$ & $\begin{array}{l}\text { FLA } \\
\left(\mathrm{cm}^{2}\right)\end{array}$ & $\begin{array}{c}\text { NT } \\
\text { (nos.) }\end{array}$ & $\begin{array}{l}\mathrm{PH} \\
(\mathrm{cm})\end{array}$ & $\begin{array}{c}\text { PL } \\
(\mathrm{cm})\end{array}$ & $\begin{array}{l}\text { PSF } \\
(\%)\end{array}$ & $\begin{array}{c}\text { HGW } \\
(\mathrm{g})\end{array}$ & $\begin{array}{c}\mathrm{GY} \\
(\mathrm{Kg} / \mathrm{ha})\end{array}$ \\
\hline IR 68888 A/ Sepidroud & $23.89 \mathrm{cde}$ & $1.35 \mathrm{~cd}$ & $23.93 \mathrm{f}$ & $26.50 \mathrm{a}$ & $94.45 \mathrm{efg}$ & $25.5 \mathrm{ab}$ & 84.98abcd & $2.65 \mathrm{abc}$ & $5969 \mathrm{a}$ \\
\hline IR 68888 A / Bejar & $23.05 \mathrm{e}$ & $1.50 \mathrm{a}$ & $25.15 \mathrm{ef}$ & $24.89 \mathrm{ab}$ & 98.78def & $26.33 \mathrm{ab}$ & $67.67 \mathrm{f}$ & $2.83 \mathrm{a}$ & $4766 f$ \\
\hline IR68888 A / IR28 & $23.05 \mathrm{de}$ & $1.44 \mathrm{abcd}$ & $24.89 \mathrm{ef}$ & $21.00 \mathrm{ab}$ & $97.58 \mathrm{defg}$ & $25.77 \mathrm{ab}$ & 84.19abcd & $2.41 \mathrm{def}$ & $4084 \mathrm{jk}$ \\
\hline $\begin{array}{l}\text { IR68888 A / IR57301- } \\
158-1 \mathrm{R}\end{array}$ & 26.77abcde & $1.43 \mathrm{bcd}$ & 28.77abcd & $20.11 \mathrm{ab}$ & $100.89 \mathrm{~cd}$ & $24.89 \mathrm{bc}$ & $79.26 \mathrm{bcde}$ & $2.28 \mathrm{efgh}$ & $3634 \mathrm{~m}$ \\
\hline $\begin{array}{l}\text { IR68888 A / IR59673- } \\
\text { 2-3-3R }\end{array}$ & $26.05 \mathrm{bcde}$ & $1.38 \mathrm{~cd}$ & $26.96 \mathrm{bcdef}$ & $21.67 \mathrm{ab}$ & $84.34 \mathrm{ik}$ & $24.50 \mathrm{bc}$ & $48.02 \mathrm{~g}$ & 2.14ghi & $2227 n$ \\
\hline IR62829 A / Sepidroud & $28.17 \mathrm{ab}$ & $1.35 \mathrm{abcd}$ & 28.52abcd & $15.00 \mathrm{~b}$ & $92.45 \mathrm{ghi}$ & $26.17 \mathrm{ab}$ & $53.05 \mathrm{~g}$ & $2.69 \mathrm{ab}$ & $2140 \mathrm{o}$ \\
\hline IR62829 A / Bejar & 26.17abcde & $1.40 \mathrm{~cd}$ & $27.47 \mathrm{bcde}$ & $25.47 \mathrm{a}$ & $94.22 \mathrm{fgh}$ & $26.17 \mathrm{ab}$ & $69.62 \mathrm{ef}$ & 2.62abc & $4681 \mathrm{~g}$ \\
\hline IR62829 A / IR28 & $27.45 \mathrm{abc}$ & $1.34 \mathrm{abcd}$ & $27.58 \mathrm{bcde}$ & $28.34 \mathrm{a}$ & $95.47 \mathrm{efg}$ & $22.94 \mathrm{c}$ & $73.52 \mathrm{def}$ & $2.56 \mathrm{bcd}$ & $5334 \mathrm{c}$ \\
\hline $\begin{array}{l}\text { IR62829 A/ IR 57301- } \\
158-1 R\end{array}$ & $27.67 \mathrm{abc}$ & $1.41 \mathrm{abcd}$ & $29.26 \mathrm{abc}$ & $30.45 \mathrm{a}$ & 89.11hij & $25.66 \mathrm{ab}$ & $77.45 \mathrm{bcdef}$ & $2.34 \mathrm{efg}$ & $5518 \mathrm{~b}$ \\
\hline $\begin{array}{l}\text { IR62829 A / IR59673- } \\
\text { 2-3-3R }\end{array}$ & $27.25 \mathrm{abcd}$ & $1.43 \mathrm{de}$ & $29.29 \mathrm{abc}$ & $29.89 \mathrm{a}$ & $82.67 \mathrm{k}$ & $26.17 \mathrm{ab}$ & $27.20 \mathrm{~h}$ & 2.21 fghi & $1796 \mathrm{p}$ \\
\hline Sepidroud & $29.95 \mathrm{a}$ & $1.33 \mathrm{ab}$ & $29.87 \mathrm{ab}$ & $22.00 \mathrm{a}$ & $99.67 \mathrm{cde}$ & $27.12 \mathrm{a}$ & $88.09 \mathrm{ab}$ & $2.65 \mathrm{abc}$ & $5135 \mathrm{~d}$ \\
\hline Bejar & $28.60 \mathrm{ab}$ & $1.47 \mathrm{abc}$ & $31.53 \mathrm{a}$ & $15.78 \mathrm{a}$ & $121.78 \mathrm{a}$ & $26.72 \mathrm{ab}$ & $92.46 \mathrm{a}$ & $2.82 \mathrm{a}$ & $4114 \mathrm{ij}$ \\
\hline IR28 & 25.72 bcde & $1.45 \mathrm{abcd}$ & $27.97 \mathrm{bcde}$ & $21.78 \mathrm{a}$ & $108.76 \mathrm{~b}$ & $26.11 \mathrm{ab}$ & 81.84abcd & $2.71 \mathrm{ab}$ & $4830 \mathrm{e}$ \\
\hline IR 57301-158-1R & $28.33 \mathrm{ab}$ & $1.41 \mathrm{abcd}$ & $29.95 \mathrm{ab}$ & $21.78 \mathrm{ab}$ & $104.0 \mathrm{bc}$ & $24.77 b c$ & $75.96 \mathrm{cdef}$ & $2.44 \mathrm{cde}$ & $4036 \mathrm{k}$ \\
\hline IR59673-2-3-3R & $16.94 \mathrm{f}$ & $1.40 \mathrm{bcd}$ & $17.79 \mathrm{~g}$ & $25.34 \mathrm{a}$ & $94.11 \mathrm{fgh}$ & $25.72 \mathrm{ab}$ & 84.98abcd & $2.07 \mathrm{hi}$ & $4457 \mathrm{~h}$ \\
\hline IR68888 B & $25.16 \mathrm{bcde}$ & $1.36 \mathrm{j}$ & $25.66 \mathrm{def}$ & $27.89 \mathrm{a}$ & 96.78defg & $26.00 \mathrm{ab}$ & $87.00 \mathrm{abc}$ & $1.61 \mathrm{j}$ & 39361 \\
\hline IR62829 B & $28.50 \mathrm{ab}$ & $1.23 \mathrm{i}$ & $26.29 \mathrm{cdef}$ & $30.23 \mathrm{a}$ & $88.23 \mathrm{ij}$ & $26.11 \mathrm{ab}$ & $67.00 \mathrm{f}$ & $2.05 \mathrm{i}$ & $4164 \mathrm{i}$ \\
\hline LSD 5\% & 2.67 & 0.07 & 2.11 & 7.04 & 3.71 & 1.46 & 8.00 & 0.15 & 41.30 \\
\hline LSD $1 \%$ & 3.66 & 0.12 & 2.90 & 9.67 & 5.10 & 2.00 & 10.99 & 0.21 & 56.73 \\
\hline
\end{tabular}

*For each trait the different letters were significantly different from each other using Duncan's multiple range test (DMRT). $F L=$ Flag leaf length; FLW=Flag leaf width; FLA=Flag leaf area; NT=Number of tillers; H=Plant Height; PL=Panicle length; $\mathrm{NSP}=$ Number of spikelets per panicle; $\mathrm{HGW}=$ Hundred grain weight; $\mathrm{PSF}=$ percent spikelet fertility; $\mathrm{GY}=\mathrm{Grain}$ yield 
Table 05: Mid-Parent heterosis (MPH) (\%) for grain yield and yield related traits in rice hybrids

\begin{tabular}{lccccccccc}
\hline Cross & FLL & FLW & FLA & PH & NT & PL & PSF & HGW & GY \\
\hline IR68888A/ Sepidroud & -18.2 & 5.5 & 9.6 & 0.5 & 1.5 & -4.2 & -14.8 & 12.8 & 28.4 \\
IR68888A/ Bejar & -17.7 & 11.0 & -15.0 & -0.6 & 8.2 & -0.3 & -13.0 & 16.3 & 15 \\
IR68888A/ IR28 & -14.9 & 7.5 & 13.0 & -0.9 & -19.0 & -0.1 & -8.0 & 1.3 & -9 \\
IR68888A/ IR 57301-158-1R & -5.7 & 8.3 & 10.8 & 0.05 & -22.7 & -2.0 & 2.3 & 1.6 & -11 \\
IR68888A/ IR59673-73-3 R & 14.6 & 4.9 & -36.8 & -7.5 & -22.0 & -5.5 & 22.4 & 3.9 & -40 \\
IR62829A / Sepidroud & 2.3 & 0.4 & -39.0 & -5.8 & -19.8 & -1.5 & -1.0 & 26.3 & -41.6 \\
IR62829A / Bejar & -2.6 & -1.0 & -22.0 & -13.8 & 16.7 & 0.5 & -3.9 & 19.2 & 16.7 \\
IR62829A / IR28 & 7.9 & -4.6 & -12.9 & -7.1 & 14.0 & -11.9 & 2.9 & 18.5 & 22 \\
IR62829A / IR 57301-158-1R & 3.5 & 1.8 & -4.9 & -11.2 & 22.6 & 1.0 & 5.3 & 15.6 & 38.9 \\
IR62829A / IR59673-73-3 R & 29.5 & 3.6 & -35.0 & -13.4 & 12.3 & 1.2 & 34.8 & 20.0 & -57 \\
\hline
\end{tabular}

FLL=Flag leaf length; FLW=Flag leaf width; FLA=Flag leaf area; NT=Number of tillers; PH=Plant Height; PL=Panicle length; NSP=Number of spikelets per panicle; $\mathrm{HGW}=$ Hundred grain weight; $\mathrm{PSF}=$ percent spikelet fertility; GY=Grain yield

Table 06: Components of genetic variance for yield and yield related traits

\begin{tabular}{lcccccccccc}
\hline \multicolumn{1}{c}{ Components of Variance } & $\begin{array}{c}\text { FLL } \\
(\mathrm{cm})\end{array}$ & $\begin{array}{c}\text { FLW } \\
(\mathrm{mm})\end{array}$ & $\begin{array}{c}\text { FLA } \\
\left(\mathrm{cm}^{2}\right)\end{array}$ & $\begin{array}{c}\text { NT } \\
(\mathrm{nos})\end{array}$ & $\begin{array}{c}\text { PH } \\
(\mathrm{cm})\end{array}$ & $\begin{array}{c}\text { PL } \\
(\mathrm{cm})\end{array}$ & $\begin{array}{c}\text { NSP } \\
(\mathrm{nos})\end{array}$ & $\begin{array}{c}\text { PSF } \\
(\%)\end{array}$ & $\begin{array}{c}\text { HGW } \\
(\mathrm{g})\end{array}$ & $\begin{array}{c}\text { GY } \\
(\mathrm{Kg} / \mathrm{ha})\end{array}$ \\
\hline Additive variance & 0.325 & 0 & 0.144 & 0.5 & 3.71 & 0.028 & 199.7 & 69.96 & 61 & 405.5 \\
Dominant variance & 0.576 & 0.002 & 3.107 & 22.7 & 6.96 & 0.624 & 19.097 & 140.2 & 120 & 1813.3 \\
$\begin{array}{l}\text { Degree of dominant } \\
\text { Share of dominant variance }\end{array}$ & 1.9 & $\infty$ & 6.5 & 30.1 & 1.94 & 6.67 & 0.43 & 2 & 1.98 & 2.98 \\
$\begin{array}{l}\text { Share of environmental } \\
\text { variance }\end{array}$ & 73.74 & 50 & 31.64 & 42.4 & 30.39 & 52.47 & 59.81 & 9.31 & 0.04 & 20.57 \\
$\begin{array}{l}\text { Heritability (\%) } \\
\text { (narrow sense) }\end{array}$ & 9.83 & 0 & 3.02 & 0.14 & 24.2 & 2 & 36.69 & 30.19 & 33.68 & 14.54 \\
\hline
\end{tabular}

FLL=Flag leaf length; FLW=Flag leaf width; FLA=Flag leaf area; NT=Number of tillers; PH=Plant Height; PL=Panicle length; NSP=Number of spikelets per panicle; $\mathrm{HGW}=$ Hundred grain weight; $\mathrm{PSF}=$ percent spikelet fertility; GY=Grain yield

Estimation of GCA effects helps in selecting parental lines with high average and low combining ability. The choice of parental lines with high GCA effects increases the probability of getting heterotic hybrids in crops (Virmani, 1994). Yuan (Yaun, 2000) reported that high yielding variety/hybrid should have larger flag leaf area. In our study we found a promising restorer line i.e. IR57301-158-1R that had positive significant GCA for FLA, FLL, PSF and GY. Therefore, the high GCA restorer lines (IR28, IR57301-158-1R and Bijar) must be utilized directly for exploiting heterosis with other CMS lines. Good combiner parents result in higher frequency of heterotic hybrids than the poor combiner parents (Virmani, 1994). Sepidroud restorer line showed promise for many traits (except GY) such as PSF and HGW can be utilized for restorer line improvement through random mating of composite populations (RMCP) or $\mathrm{R} \times \mathrm{R}$ crossing programs to evolve improved restorer lines i.e. 2, 3, 14, in rice, heterotic hybrids can be obtained by crossing parents with high $\times$ low and high $\times$ high GCA effects (Kumar and Saini,1981; Pradhan et al.,2006 ; Rahman, et al.,1981; Ranganathan et al.,1973; Rao, et al.,1980; Singh and Nanda,1976). Even heterosis has been reported for average $\times$ poor general combiners (Kumar and Saini, 1981). However, in this study we found that low $x$ high GCA effects resulted in heterotic hybrid combination like the IR62829A/IR57301- 
158-1R. Interestingly, the SCA effect for this combination was non- significant for all traits except for grain yield. Similar findings in japonica rice and maize hybrids had been earlier reported (Moon, 1994; Sughroue and Hallauer, 1997). However, (Pradhan et al.,1990) found in majority of crosses high SCA for grain yield and attributed it to dominance and epistasis gene action.

In this study, low $\times$ low GCA gave the best heterotic combination i.e.IR68888A/ 'Sepidroud'. However, the line 'Sepidroud' showed non-significant GCA effects for grain yield, NSP, PL, PH, FLL, FLW and FLA while significant but positive GCA effects for NT, PSF, HGW with tester IR68888A. Recently in a study a low GCA $\times$ low GCA combination resulted in the most heterotic hybrid combination. It may be possible to get heterotic combinations from low GCA parents and therefore it will be unwise to discard the low GCA types from the hybrid breeding materials. It indirectly implies that there exist different types of negative blocks in yield biochemical pathways in such low GCA lines but when two such low GCA types come in hybrid combination. They are easily overcome by their effective complementation resulting in heterotic combinations. However, the probability to detect one such hybrid combinations is remote and low. Singh and Nanda reported high SCA values of certain crosses derived from poor combining parents. Pradhan et al., (2006) also showed a few crosses showing low/low general combiners with high SCA, suggesting the epistatic gene action due to the genetic diversity in the form of heterozygous loci. At IRRI the combining ability studies showed the crosses showing high SCA effects involved parents with high/ low or low/high GCA (in 30\% cases), low/ low GCA or low/ average GCA (in 16\% cases). High/high GCA and high/average GCA parents resulted in high SCA effects in 12 and $15 \%$ crosses, respectively (Virmani, 1997; Young, 1987). In other words, the heterotic combinations must be selected based on significant SCA effects for grain yield and its per se performance over the standard check variety for both yield and grain quality.

Two hybrids i.e. IR62829A/IR57301-158-1R and IR68888A/Sepidroud were found to be highly significant for grain yield based on the SCA effects and had 38.9 and 28.4\% MPH for yield with corresponding actual yield levels of 5518 and $5969 \mathrm{~kg} /$ ha respectively (Table 04 and 05). Such hybrid combinations will be utilized for exploitation of hybrid vigour after assessing their cooking quality features. It is always better to identify superior hybrid combinations on the basis of significant SCA effects and after examining the heterosis values (Virmani, 1997).

The results indicated a presence of dominance for yield as reported by several researchers ( Maurya and Singh, 1977; Pradhan, and et al., 2006 ; Ranganathan et al., 1993). Like yield and yield components, both GCA and /or SCA effects were important in different crosses in the inheritance of these traits (Virmani, 1997). Pradhan et al., (2006) showed the importance of non- additive gene action in their expression and indicated the good prospects for the exploitation of non-additive genetic variation for grain yield and its component characters through hybrid breeding. Virmani et al., (1997) the non-additive variance share for formation of leaf area, panicle length, 100-grain weight fertility percentage, plant height and grain yield were much more. In this study, we observed predominance of dominant gene action for traits like GY, HGW, PSF, PL, NT, FLA, FLW while NSP, FLL showed high heritability. In majority of cases, high SCA effects for yield were attributable to dominance and epistatic gene interaction and only a few cases attributable to additive/additive interaction (Young, 1987). Predominance of dominant gene action in this study for most of the yield and yield related traits such as GY, HGW, PSF, PL, NT, FLA, 
and FLW strongly favors heterosis breeding. The predominance of dominant gene action for a majority of the yield related traits had been earlier reported by several researchers ( Dwivedi et al., 1999; Peng and Virmani, 1990; Satyanarayana, et al., 2000; Sharivastava and Sheshu, 1983; Virmani et al. 1997) Selection of parents with good combining ability for selective traits that could complement each other favourably in the hybrids would be a desirable approach to breed better hybrid combinations. However, this study clearly shows that the heterosis breeding must be strongly pursued for exploiting the yield advantage over the inbred rice varieties.

\section{REFERENCES}

Ali, A. J. (2006). A big step towards achieving self sufficiency through hybrid technology for Iran In: Progress Report of Hybrid and Molecular Rice Breeding Program of Iran (HMRBPI). Rice Research Institute of Iran, Rasht, Iran.

Allahgholipour, M. and Ali, A. J. (2006). Gene action and combining ability for grain yield and its components in rice. Journal of Sustainable Agriculture. 28: 39-53.

Can, N.D., Nakamura, S. and Yoshida, T. (1997). Combining ability and genotype $\times$ environment interaction in early maturity grain sorghum for summer seeding. Japanese Journal of Crop Science. 66: 698-705.

Dwivedi, D.K., Pandey, M.P., Pandey, S.K. and Li, R. (1999). Combining ability over environments in rice involving indica and tropical japonica lines. Oryza. 36:101-107.

Gomez, K.A. and Gomez, A. A. (1984). Statistical procedures for agricultural research. John Wiley and Sons, Inc. New York.

Gravois, K.A. and McNew, R.W. (1993). Combining ability and heterosis in U.S. southern long-grain rice. Crop Science. 33: 83-86

IRRI. (1996). Standard evaluation system. Manila, Philippines.

Kempthorne, O. (1957). An introduction of genetic statistics. John Wiley \& Sons, Inc. New York.

Kumar, I. and Saini, S.S. (1981). Diallel analysis in rice. Combining ability for various quantitative characters. Genetic Agricultural. 35: 243-252.

Maurya, D.M. and Singh, D.P. (1997). Combining ability in rice for yield and fitness. Indian Journal of Agricultural Sciences. 47: 65-70.

Moon, H.P. Heu, M. H. and Kim, C. H. (1994). Hybrid rice research in the Republic of Korea. In. Virmani S.S. (Ed.) Hybrid Rice Technology: new developments and future prospects. International Rice Research Institute, Manila, Philippines. P: 217-226.

Peng, J.Y. and Virmani, S.S. (1990). Combining ability for yield and four related traits in relation to breeding in rice. Oryza. 27:1-10.

Pradhan, S.K. Bose, L.K. Meher, J. (2006). Studies on gene action and combining ability analysis in Basmati rice. Journal of Central European Agriculture. 7: 267-272. 
Rahman, M. Patwary, A.K. and Miah, A.J. (1993). Combining ability in rice. Indian Journal of Agricultural Science. 51:543-546.

Ramalingam, J. Vivekanaudan, P. and Vanniarajan, C. (1981). Combining ability analysis in lowland early rice. Crop Research. 6:228-233.

Ranganathan, T.B. Menon, P. M. and Sreerangaswamy, S.R. (1973). Combining ability of earliness and yield in dwarf varieties. Madras Agricultural Journal. 60: 1134-1138.

Rao, A.V., Krishna, S.T. and Prasad, A.S.R. (1980). Combining ability analysis in rice. Indian Journal of Agricultural Science. 50:193-197.

Satyanarayana, P.V. Reddy, M.S.S. Kumar, I. and Madhuri, J. (2000). Combining ability studies on yield and yield components in rice. Oryza. . 57:22-25.

Shrivastava, M.N. and Sheshu, D.V. (1983). Combining ability for yield and associated characters in rice. Crop Science. 23:741-744.

Siddiq, E.A. Jachuck, P.J. Mahadevappa, M. Zaman, F.U. Vijayakumar, R. Vidhyachandra, B. Sidhu, G.S. Kumar, I. Prasad, M.N. Rangaswamy, M. Pandey, M.P. Panwar, D.V.S. and Ahmed, I. (1994). Hybrid rice research in India. In Virmani S.S. (Ed.) Hybrid Rice Technology: new developments and future prospects. International Rice Research Institute, Manila, Philippines. P: 157-171.

Singh, D.P. and Nanda, J.S. (1976). Combining ability and heterosis in rice. Indian Journal of Genetics.; 36:10-15.

Sughroue, J.R. and Hallauer, A.R (1997). Analysis of the diallel mating design for maize inbred lines. Crop Science. 37: 400-405.

Tran, D.V. (2002). Hybrid rice for food security: recent progress and large- scale production issues. In: Proceedings of the workshop on policy support for rapid adoption of hybrid rice on large scale production in Asia, Hanoi, Vietnam, 22-23 May 2001. Ministry of Agriculture and Rural development, Vietnam, International Rice Research Institute, Philippines; Food and Agriculture organization of the United Nations, Rome. P: 17-35.

Virmani, S.S. (1994). Heterosis and hybrid rice breeding- Monographs on Theoretical and Applied Genetics 22, Springer-Verlag, Berlin, Heidelberg.

Virmani, S.S. Viraktamath, B.C. Casal, C.L. Toledo, R.S. Lopez, M.T. and Manalo, J.O. (1997). Hybrid Rice Breeding Manual, International Rice Research Institute, Philippines.

Young, J.B. (1987). Heterosis and combining ability over environments in relation to hybrid rice breeding. Ph.D. Thesis, University of the Philippines, Los Banos, Philippines.

Yuan L.P. (1998). Hybrid rice breeding in China. In Virmani S.S. Siddiq E.A., Muralidharan K. (Eds.) Advances in hybrid Rice Technology. International Rice Research Institute, Manila, Philippines. P: 27-33.

Yuan L.P. (2000). Super hybrid rice. Hunan Agricultural Science and Technology Newsletter. 1: 8-12. 